\title{
The Effect of Mangiferin on Improving Endothelial Dysfunction by Inhibiting Endoplasmic Reticulum Stress to Alleviate Mitochondrial Fission
}

\author{
Yang Chen ${ }^{1,2}$, Yu-peng Chen ${ }^{1,2}$, Bao-lin Liu ${ }^{1,2}$, Kang Liu ${ }^{1,2}$, Fang Huang ${ }^{1,2, *}$, Zhi-xia Qiu 1, 2, * \\ ${ }^{1}$ Department of Pharmacology of Chinese Materia Medica, China Pharmaceutical University, Nanjing, China \\ ${ }^{2}$ Jiangsu Key Laboratory of TCM Evaluation and Translational Research, China Pharmaceutical University, Nanjing, China
}

\section{Email address:}

fanghuangcpu@163.com (Fang Huang)

*Corresponding author

\section{To cite this article:}

Yang Chen, Yu-peng Chen, Bao-lin Liu, Kang Liu, Fang Huang, Zhi-xia Qiu. The Effect of Mangiferin on Improving Endothelial Dysfunction by Inhibiting Endoplasmic Reticulum Stress to Alleviate Mitochondrial Fission. American Journal of Clinical and Experimental Medicine. Vol. 5, No. 5, 2017, pp. 162-172. doi: 10.11648/j.ajcem.20170505.12

Received: July 23, 2017; Accepted: August 16, 2017; Published: August 31, 2017

\begin{abstract}
Microvascular and macrovascular diseases are important complications of metabolic diseases and affect the normal functioning of the human cardiovascular system. Endothelial dysfunction is the basic pathological tache of vascular diseases. This study aims to find out whether mangiferin can relieve endothelial dysfunction by inhibiting mitochondrial fission induced by endoplasmic reticulum stress. After being stimulated by palmitate, the expression of endoplasmic reticulum stress-related proteins Bip, p-PERK (RNA-like endoplasmic reticulum kinase) increased significantly. The endoplasmic reticulum stress was effectively inhibited after the treatment with mangiferin and the inhibition diminished after the knockout of AMPK (AMP-activated protein kinase) through AMPK siRNA interference, which demonstrated that AMPK was a key point for mangiferin action to exert its therapeutic effects. Under endoplasmic reticulum stress conditions, the influx of calcium ions from the endoplasmic reticulum into cytoplasm may lead to mitochondrial calcium ions overload and a large increase in mitochondrial ROS. As a result, mitochondrial fission was further promoted and apoptosis was induced. Mangiferin effectively improved the endothelial function by activating AMPK to inhibit the endoplasmic reticulum stress, maintaining the calcium homeostasis in mitochondria while inhibiting apoptosis by diminishing mitochondrial fission and ROS (Reactive Oxygen Specie) generation. In conclusion, mangiferin could maintain $\mathrm{Ca}^{2+}$ homeostasis in mitochondria under ER stress conditions, attenuate cell apoptosis induced by mitochondrial fission and ROS generation, and effectively improve the endothelial function by activating AMPK to inhibit endoplasmic reticulum stress.
\end{abstract}

Keywords: Mangiferin, Endoplasmic Reticulum Stress, Mitochondrial Dysfunction, Endothelial Dysfunction

\section{Introduction}

Obesity and diabetes are accompanied with an increasing risk of developing cardiovascular disease[1-4], since glucose and lipid metabolism disorder can stimulate vascular endothelial cells to induce a stress response, to which endoplasmic reticulum (ER) and mitochondria are exquisitely sensitive [2-5]. If such an external stimulation is prolonged, endoplasmic cells cannot maintain the normal physiological functions by self-regulation and pathological stress occurs as a result. Endothelial dysfunction has been observed in patients with chronic metabolic diseases, such as diabetes, cardiovascular disease and atherosclerosis [6].
In eukaryotic cells, ER is a vital apparatus for protein biogenesis, folding and translocation, while also participating in cell signaling. When excessive unfolded proteins exist in the ER lumen, the stability of the ER will be destroyed and Endoplasmic Reticulum stress (ER stress) occurs. In such a case, Unfolded Protein Response (UPR) can be triggered to restore proteostasis of ER. There are three UPR target transducers in the ER membrane, namely protein kinase RNA-like endoplasmic reticulum kinase (PERK), IRE1, and Activating Transcription Factor 6 (ATF6) and their cascade. The canonical UPR aims to increase protein folding and decrease biogenesis and secretion so as to attenuate stress. However, if the stress cannot be sufficiently 
neutralized, the endothelial cells will be in a sustained stress status $[7,8] \cdot \mathrm{Ca}^{2+}$ also locals in the ER $\left(\left[\mathrm{Ca}^{2+}\right]_{\mathrm{ER}}\right)$, when cells undergoes the ER stress and $\left[\mathrm{Ca}^{2+}\right]_{\mathrm{ER}}$ will exude to the cytosolic, which may trigger inflammation, apoptosis, and oxidative stress. Increasing evidence has suggested that ER stress is responsible for endothelial dysfunction [9].

Generally, mitochondria are "energy-supplement" organelle, playing an important role in endothelial homeostasis [10]. It participates in cell survival and death by regulating their morphology through fission and fusion proteins, and is involved in cell mitosis, embryonic development, and energy metabolism. Furthermore, it maintains cellular metabolic processes including enzyme reactions through oxidative phosphorylation, which is vital for cell death and survival [11-13]. There is increasing evidence revealing the close relationship between endothelial dysfunction and perturbative mitochondrial morphology [14].

The mitochondria play a critical role in the endothelial cells because of ROS generation and calcium homeostasis maintaining. ER and mitochondria interact with each other not only in structure but also in function [13]. Increasing evidence has suggested that mitochondria morphology and function have important roles in endothelial dysfunction [15]. Under stress, $\left[\mathrm{Ca}^{2+}\right]$ ER transfers to the cytoplasm and the mitochondrial uptake the $\mathrm{Ca}^{2+}$ regulates the level of $\mathrm{Ca}^{2+}$ in cells during routine functioning. In the event of potential mitochondrial membrane collapse, ROS generation will be induced due to $\mathrm{Ca}^{2+}$ overload $[13,16]$. Meanwhile, excessive $\mathrm{Ca}^{2+}$ in cytoplasm can increase calcineurin concentration and facilitate Drp1 (dynamin-related protein 1) dephosphorylation and transfer to the mitochondrial outer membrane. Afterwards, mitochondrial fragmentation increases and cytochorome $\mathrm{c}$ is released to trigger apoptosis [17, 18]. More recent data, however, have suggested endothelial dysfunction is related to mitochondrial dysfunction.

Mangiferin (MGF) is one of the main constituents in common anemarrhena, a traditional medicine, for treating diabetes [19-21] Recently, it has been reported that MGF can attenuate high-fat diet (HFD)-induced metabolism syndrome through activating AMP-actived protein Kinase (AMPK) for abnormal lipid metabolism regulation, anti-inflammation, and insulin resistance improvement [19]. A recent study demonstrates that MGF can ameliorate endothelial dysfunction by regulating AMPK to inhibit the inflammation in ER stress [20]. A large body of evidence has reported that MGF can regulate AMPK activation, which is sensitive to the ratio of AMP to ATP. AMP/ATP plays a vital role in intracellular homeostasis and it has been well documented that activation of AMPK $\alpha$ can inhibit ROS generation to attenuate mitochondrial fission and ameliorate apoptosis of endothelial cells undergoing diabetes [22]. However, a recent report demonstrated that AMPK directly participates in mitochondrial fission and activated AMPK can also facilitate mitochondrial fission [23]. However, the effect MGF has in preventing or significantly reducing endothelial dysfunction with ER stress and the mechanism by which it regulates AMPK activation in the mitochondrial fission process is still unclear. Therefore, we designed the relevant experiments to explore this problem and enhance our understanding of the process by which MGF facilitates and regulates cellular interactions to improve conditions resulting from endothelial dysfunction.

The mechanism by which MGF regulates endothelial dysfunction is related to ER stress and mitochondria fission. Our preliminary results showed that MGF could activate AMPK to attenuate ER stress, while AMPK also facilitated mitochondria fission. Therefore the relationship between ER stress and mitochondria fission requires further explored. The results showed that MGF could ameliorate mitochondrial fission and $\left[\mathrm{Ca}^{2+}\right]_{\text {mito }}$ overload induced by ER stress. MGF could inhibit ER stress through activating AMPK, so as to maintain mitochondrial homeostasis and attenuate cell apoptosis. We hypothesized ER function and morphology homeostasis were responsible for the mitochondrial function and morphology homeostasis.

\section{Materials and Methods}

\subsection{Reagents}

Mangiferin (MGF) was obtained from Nanjing Zelang Medical Technological Co., Ltd (Nanjing, China). AICA riboside (AICAR) was purchased from Beyotime Institute of Biotechnology (Shanghai, China). Tauroursodeoxycholic acid (TUDCA) was procured from Sigma (St Louis, MO, USA). Mito-TEMPOL was obtained from Abcam (Cambridge, MA, USA). These reagents were dissolved in dimethyl sulfoxide (DMSO) before use and the final working concentration was lower than $0.1 \%(\mathrm{v} / \mathrm{v})$. Palmitate (PA, Sinopharm Chemical Rwagent wq Co., Ltd. Shanghai, China) was dissolved in anhydrous ethanol to prepare a $200 \mathrm{mM}$ stock solution and then was further diluted with medium containing $10 \%$ bovine serum albumin (BSA) at the ratio of $1: 19$ to obtain a concentration of $10 \mathrm{mM}$ prior to experimental application. The following items were purchased from the cited commercial sources: Tunicamycin (Tun), Acetylcholine (Ach), Sigma (St Louis, MO, USA); phenylephrine (TCI, Shanghai, China); anti-phospho-AMPK $\alpha$ (T172) (2531 s), anti-AMPK $\alpha$ (2532 s), anti-PERK (\#3192), Cell Signaling Technology (Beverly, MA, USA); anti-eIF2 $\alpha($ ab26197), anti-phosphoeIF2 $\alpha($ ab32157), anti-ATF4(ab184909), anti-Drp1(ab184247), anti-phospho-Drp1(ab193216), Abcam (Cambridge, MA, USA); anti-GAPDH(AP0063), Goat Anti-Rabbit IgG(BS13278), Bioworld Technology(St. Paul, MN, USA); AMPK $\alpha 1 / 2$ siRNA(sc-45312), Control siRNA(sc-37007), siRNA transfection(sc-29528), Santa Cruz Biotechnology, Inc. (Santa Cruz, CA, USA).

\subsection{Animals}

Sprague-Dawley male rats (180-220 g) and ICR male mice (18-22 g) were obtained from Laboratory Animal Center of Yangzhou University. These animals were cared according to the Provisions and General Recommendation of Chinese Experimental Animals Administration Legislation. These animals were maintained a constant temperature $\left(22 \pm 2^{\circ} \mathrm{C}\right)$, unlimited standard diet and water, in a $12 \mathrm{~h}$ light-dark cycle. This study was performed in compliance with the guide for the Care and Use of Laboratory Animals (2011 version; National Institutes of Health) and approved by the Animal Ethics 
Committee of the School of Chinese Materia Medica at China Pharmaceutical University.

\subsection{Cell Preparation and Culture}

Rat aortic endothelial cells (RAECs) were prepared as previously described [22]. In summary, RAECs were isolated from rat thoracic aortas explants using collagenase I and cultured in Dulbecco's Minimum Essential Medium (DMEM) supplemented with $20 \%$ fetal bovine serum (FBS) until cellular division was observed from the endothelial tissue being maintained at $37^{\circ} \mathrm{C}$ in a humidified atmosphere of $5 \% \mathrm{CO}_{2}$. Passages 3 to 5 of the RAECs were available for the experiments.

Human umbilical vein endothelial cells (HUVECs, provided by the cell bank of the Chinese Academy of Sciences, Shanghai, China) were cultured in the RPMI 1640 medium containing $10 \%(\mathrm{v} / \mathrm{v})$ fetal bovine serum (FBS), $100 \mu \mathrm{g} \cdot \mathrm{mL}-1$ streptomycin and $100 \mathrm{U} \cdot \mathrm{mL}-1$ penicillin at $37^{\circ} \mathrm{C}$ in a $5 \% \mathrm{CO}_{2}$ atmosphere. After $80 \%$ confluence, cells were washed twice with PBS and switched to a serum-free medium for 2-4 $\mathrm{h}$ before further experiments were conducted [22].

\subsection{Assay of ROS Production and NO}

RAECs were cultured in a 48-well plate, as cells were cultured to $80 \%$ confluency and then treated with MGF (10 $\mathrm{mM})$, AICAR $(500 \mu \mathrm{M})$, mito-TEMPO $(20 \mu \mathrm{M}), \mathrm{Ru} 360(10$ $\mu \mathrm{M}), \quad \operatorname{RuR}(10 \mu \mathrm{M}), \mathrm{Xe} C(10 \mu \mathrm{M})$ in the presence or absence of PA $(100 \mu \mathrm{M})$ and Tun $(5 \mathrm{ng} / \mathrm{mL})$ for $24 \mathrm{~h}$ and $8 \mathrm{~h}$ respectively. After treatment, cells were washed with PBS twice, and then kept in the dark with ROS specific fluorescent probe dye dihydroethidium (Beyotime Institute of Biotechnology, Shanghai, China) for $30 \mathrm{~min}$ and DAPI (Sigma, St. Louis, MO, USA) for $5 \mathrm{~min}$ at $37^{\circ} \mathrm{C}$. After being washed, cells were fixed in $4 \%$ paraformaldehyde $(\mathrm{v} / \mathrm{v})$ for $5 \mathrm{~min}$ at $4^{\circ} \mathrm{C}$, and $\mathrm{ROS}$ production was viewed by a fluorescence microscope or measured using a microplate reader at an excitation wavelength of $488 \mathrm{~nm}$ and an emission wavelength of $525 \mathrm{~nm}$.

For the detection of NO, RAECs were cultured to $80 \%$ confluency and then pretreated with MGF (10 mM), AICAR $(500 \mu \mathrm{M})$, TUDCA $(100 \mu \mathrm{M})$ for $30 \mathrm{~min}$, and then incubated with PA $(100 \mu \mathrm{M})$ and Tun $(5 \mathrm{ng} / \mathrm{mL})$ for $24 \mathrm{~h}$ and $8 \mathrm{~h}$ respectively. After treatment, cells were washed with PBS and incubated with NO-specific fluorescent dye DAF-FMDA (Beyotime, Shanghai, China) at $37^{\circ} \mathrm{C}$, in the dark for $20 \mathrm{~min}$. For the observation of $\mathrm{NO}$ in the vascular endothelium with HFD-fed mice, isolated thoracic aortas were then incubated with $10 \mu \mathrm{m}$ ACh for $30 \mathrm{~min}$ and then stained with DAF-FMDA and DAPI. After being washed with PBS twice, RAECs and thoracic aortas were detected with a fluorescence microscope.

\subsection{Mitochondria Fission and Mitochondrial Membrane Potential ( $\triangle \psi m$ ) Analysis}

For mitochondrial fission assay, RAECs were pretreated with MGF, TUDCA and RuR for 30 min, and then incubated with PA and Tun for $24 \mathrm{~h}$ and $8 \mathrm{~h}$ respectively. After the treatment and being washed by PBS twice, RAECs were fixed in Mito Tracer Red CMX Rox $(200 \mathrm{nmol} / \mathrm{L})$ for $30 \mathrm{~min}$ at $37^{\circ} \mathrm{C}$. The structure of mitochondria was viewed by confocal scanning microscopy. The results were evaluated for mitochondrial morphology from 90 cells in three independent experiments.

Mitochondrial membrane potential $(\Delta \psi \mathrm{m})$ was examined by TMRE staining using confocal microscopy. After treatment, RAECs cells were incubated with potentiometric dye TMRE $(500 \mathrm{nM})$ for $30 \mathrm{~min}$ at $37^{\circ} \mathrm{C}$. The results were determined by single-cell fluorescence intensities from 30 cells in every treatment group.

\subsection{Assay of Apoptosis, Mitochondrial $\mathrm{Ca}^{2+}$ and Caspase-3}

Confluent RAECs were pretreated with MGF, TUDCA for $30 \mathrm{~min}$, and then incubated with PA for $24 \mathrm{~h}$. After incubation, cells were collected and stained with Annexin V-FITC Apoptosis Detection Kit (Key GEN Biotech Co., Ltd. Nanjing, China). According to manufacturer's instructions, cellular fluorescence was imaged using flow cytometry analysis with a FACS Calibur Flow Cytometer (BD Biosciences, USA).

Confluent RAECs were pre-treated with MGF and TUDCA for $30 \mathrm{~min}$ respectively, and then fixed in PA and Tun for $24 \mathrm{~h}$ and $8 \mathrm{~h}$ respectively. After treatment, cells were washed by PBS twice. In order to detect mitochondrial $\mathrm{Ca}^{2+}$, cells were lysed by commercial Kit to collect mitochondria. Then cells were washed by PBS twice and O-cresolphthalein complexone was used to assay the concentration of $\mathrm{Ca}^{2+}$ in mitochondria.

As cells reached an $80 \%$ degree of fusion while being cultured in a 6-well plate, they were treated with MGF, TUDCA, anti-IL-1 $\beta$ for $30 \mathrm{~min}$, and then fixed in PA and Tun for $24 \mathrm{~h}$ or $8 \mathrm{~h}$ respectively. After treatment, collected cells were lysed in ice-cold lysis buffer to extract protein by centrifugation at $12000 \mathrm{~g}, 4^{\circ} \mathrm{C}, 15 \mathrm{~min}$. Caspase- 3 activity was determined by a commercial kit (Beyotime, Shanghai, China).

\subsection{The SIRNA-Mediated Gene Silencing}

To specifically silence AMPK gene expression, HUVECs were respectively transfected with human specific AMPK a1/2 (sc-45312, Santa Cruz Biotechnology) duplexes small interfering RNA (siRNA) or control siRNA (sc-37007) by siRNA transfection reagent (sc-29528). After cells had been transfected for $7 \mathrm{~h}$, they were cultured in a medium for $48 \mathrm{~h}$ and then switched to a serum-free medium for $2 \mathrm{~h}$. The cells were pretreated with MGF, TUDCA for $30 \mathrm{~min}$, and incubated with PA and Tun for $24 \mathrm{~h}$ and $8 \mathrm{~h}$ respectively and the protein expression was examined by western blot.

\subsection{Western Blot Assay and Immunohistochemistry}

HUVECs or RAECs were lysed in an ice-cold RIPA buffer containing $1 \mathrm{mM}$ PMSF for $45 \mathrm{~min}$ separately, then underwent centrifugation at $12000 \mathrm{~g}$ for $20 \mathrm{~min}$ at $4{ }^{\circ} \mathrm{C}$, and the concentration was quantified using Bicinchoninic Acid Protein Assay Kit (Biosky Biotechnology Corporation, Nanjing, China). An equal amount of protein was electrophoresed on SDS-PAGE, transferred to polyvinylidene difluoride (PVDF) membranes, and then blocked at room temperature for $2 \mathrm{~h}$. For immunoblotting, the primary 
antibodies were used respectively at $4^{\circ} \mathrm{C}$ overnight, followed by incubation with the secondary antibody at room temperature for $2 \mathrm{~h}$. An enhanced ECL Kit was used to detect signals and Image-Pro Plus 6.0 (IPP 6.0) software was employed for quantitative analysis by densitometry.

The thoracic aortas were isolated from the mice and then cultured in a 6-well plate and then the thoracic aortas were washed with PBS and incubated with $4 \%$ paraformaldehyde for $24 \mathrm{~h}$, dehydrated and embedded in paraffin, then cut into 4 $\mu \mathrm{M}$ thickness and affixed onto slides, followed by deparaffinization and rehydration. After rehydration, antigens were retrieved by microwave heat. The slides were then blocked with $3 \%$ peroxide-methanol for $25 \mathrm{~min}$ at room temperature. The slides were incubated with primary antibody overnight at $4{ }^{\circ} \mathrm{C}$ followed by incubation with HRP-conjugated secondary antibody for $50 \mathrm{~min}$ at room temperature. The sections were stained with diaminobenzidine (DAB) for $10 \mathrm{~min}$ in the dark and counterstained using hematoxylin nuclear stain and finally detected the microscope.

\subsection{Endothelium-Dependent Relaxation Assay}

The rat thoracic aortas were isolated as previously described [22]. In brief, the thoracic aortas were removed from the rats and incubated in an ice-cold Krebs-Henseleit
(K-H) solution to remove fat and connective tissues. The aortas were cut into $5 \mathrm{~mm}$ rings, and then the rings were incubated in the $\mathrm{K}-\mathrm{H}$ solution and maintained at $37^{\circ} \mathrm{C}$ with $95 \% \mathrm{O}_{2}$ and $5 \% \mathrm{CO}_{2}$. The aorta rings were exposed to $60 \mathrm{mM}$ $\mathrm{KCl}$ to assay the contractile activity. The functionality of vascular endothelium was confirmed by the ability of relaxation (over $80 \%$ ) in response to $10 \mu \mathrm{M}$ Ach, after the rings were pre-contracted with $1 \mu \mathrm{M}$ phenylephrine (TCI, Shanghai, China). Each aorta ring was pretreated with MGF and TUDCA respectively for $30 \mathrm{~min}$, and then incubated with PA and Tun for $30 \mathrm{~min}$ respectively. Then they were washed three times using K-H solution and pre-contracted with $1 \mu \mathrm{M}$ phenylephrine. Finally, the rings were cumulatively treated with Ach $(0.001-10 \mu \mathrm{M})$ to test the endothelium-dependent relaxation function. The relaxation was expressed as a percentage of the phenylephrine-induced contraction.

\subsection{Statistical Analysis}

All results were expressed as means \pm SD (standard deviation). Data were analyzed by using two-tailed t-test or one-way ANOVA test with Student-Newman-Keuls test for comparison of two groups. A value of $\mathrm{p}<0.05$ was considered statistically significant.
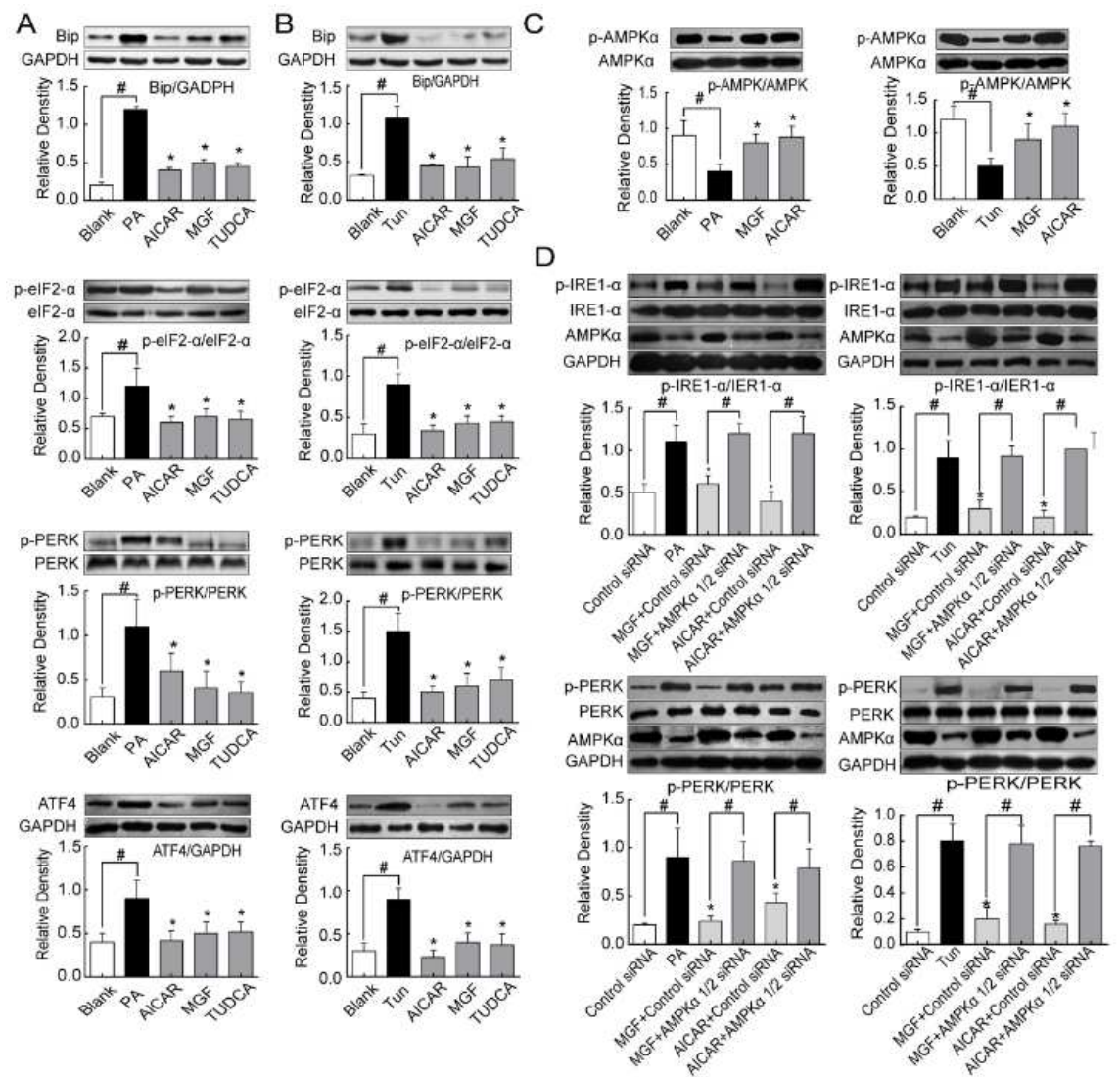

Figure 1. MGF and AICAR prevented ER stress through activating AMPK. (A): primary rat aorta endothelial cells (RAECs) were pretreated with MGF, AICAR and TUDCA respectively at given concentrations, and then incubated with palmitate (PA) for 24 ; Bip, p-PERK, p-eIF2- $\alpha$ and ATF4 protein expression in RAECs were determined with Western blot. (B): RAECs were pretreated with MGF, AICAR and TUDCA respectively, and then incubated with Tun for 8 h. Bip, p-PERK, p-eIF2- $\alpha$ and ATF4 protein expression in RAECs were determined with Western blot. (C): HUVECs were incubated with MGF and AICAR separately in the presence of PA for $24 \mathrm{~h}$ and Tun for $8 \mathrm{~h}$. (D): MGF and AICAR incubated with PA for $24 \mathrm{~h}$ or Tun for $8 \mathrm{~h}$ in HUVECs which were transfected with AMPKal/2 or control scrambled siRNAs. AMPK, IRE1- $\alpha$ and PERK phosphorylation were determined by Western blot. The results were expressed as the mean \pm SD of three independent experiments. ${ }^{*} p<0.05$ : indicated group vs PA or Tun only treatment; \#p<0.05: PA or Tun treatment vs untreated control. 


\section{Results}

\subsection{MGF and AICAR Prevented ER Stress Through Activating AMPK}

RAECs stimulated by PA could improve the expression of Bip, p-PERK, p-eIF2 $\alpha$, and ATF4, which indicated cells were undergoing ER stress. MGF could ameliorate ER stress as the AMPK activator AICAR (figure 1A). Tun can influence in protein synthesis to induce the ER dysfunction and trigger ER stress. MGF and AICAR prevented cells from ER stress

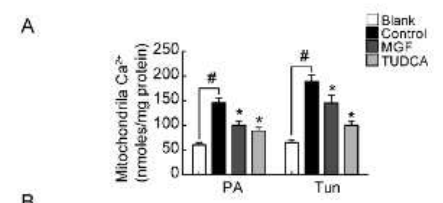

B

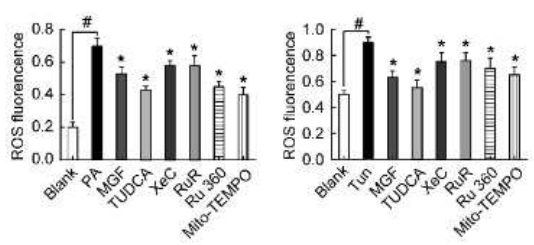

D

Mitochondrial Membrane Polential
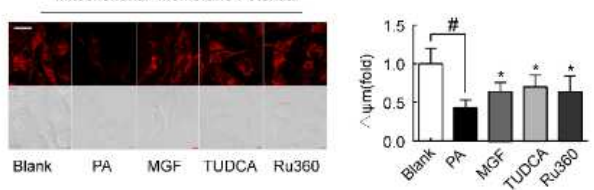

Mito-TEMPO
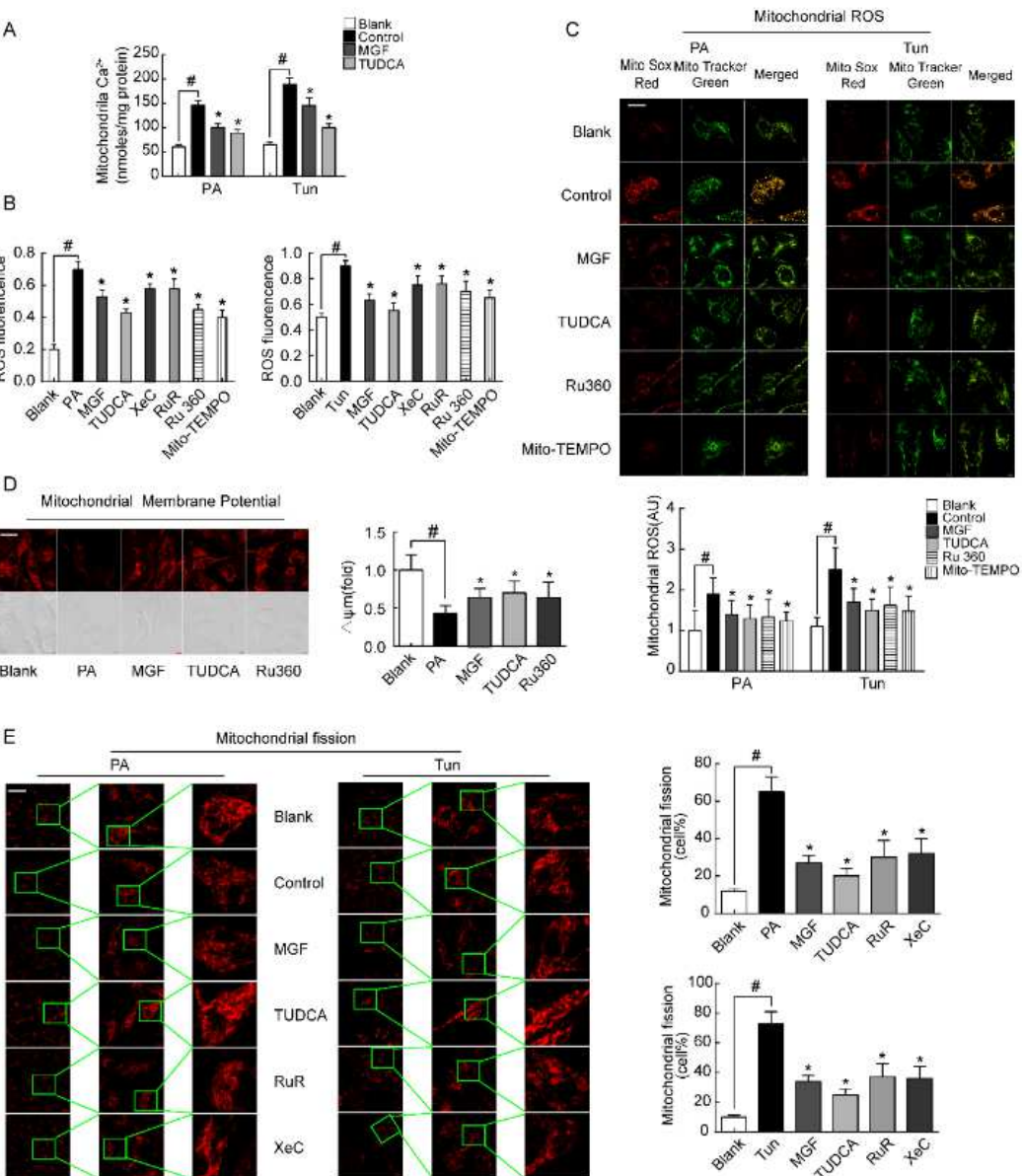

Figure 2. MGF attenuated mitochondrial $\mathrm{Ca}^{2+}$ overload. RAECs were incubated with MGF and AICAR separately in the presence of PA and Tun for 24 h and 8 $h$ respectively; $(A)$ : direct measurement of total $\mathrm{Ca}^{2+}$ content in mitochondria, isolated from $R A E C s$. Absolute Ca ${ }^{2+}$ content (expressed as nmoles/mg protein) was determined using the o-cresolphthalein complexone assay. RAECs were pretreated with MGF (10 $\mu M) T U D C A(100 \mu M), X e C(10 \mu M)$, RuR (10 $\mu M)$, Ru $360(10$ $\mu M)$, Mito-TEMPO $(20 \mu M)$ and then incubated with PA for 24 h or Tun for $8 \mathrm{~h}$. (B): ROS production in RAECs was measured with a fluorescence microplate reader. (C): mitochondrial ROS levels were measured by Mito Sox Red (red) using confocal microscopy. Mito Sox Red is colocalized with Mito Tracker Green. Scale bar, $5 \mu m$. (D): RAECs were stimulated with PA for $24 \mathrm{~h}$ in the presence of MGF, TUDCA and Ru 360, and $\triangle 4 m$ was viewed by TMRE labeling with confocal microscopy. Bar Scale $=5 \mu \mathrm{m}$. (E): RAECs were pretreated with MGF, TUDCA, RuR and XeC separately, and then incubated with PA and Tun for 24 h and $8 \mathrm{~h}$ respectively. Mitochondrial fission was detected by Mito Tracker Red CMX Ros with confocal microscopy and represented by the percentage of fragmented mitochondria. Scale bar, $5 \mu \mathrm{m}$. The results were expressed as the mean $\pm S D$ of three independent experiments. *p<0.05: indicated group vs PA or Tun only treatment; \#p<0.05: PA or Tun treatment vs untreated control.

\subsection{MGF Attenuated Mitochondrial $\mathrm{Ca}^{2+}$ Overload}

Under ER stress, mitochondria will take in the excessive $\mathrm{Ca}^{2+}$ resulting in a significantly large amount of $\mathrm{Ca}^{2+}$ uptake in the mitochondria. This phenomenon, known as mitochondrial $\mathrm{Ca}^{2+}$ overload, was stimulated by PA or Tun. MGF can significantly reduce the $\mathrm{Ca}^{2+}$ concentration as an ER stress inhibitor TUDCA (Figure 2A). As cytoplasm and mitochondria were responsible for ROS generation, we treated RAECs with MGF, TUDCA, $\mathrm{ER} \mathrm{Ca}^{2+}$ inhibitor RuR and $\mathrm{XeC}$, mitochondrial $\mathrm{Ca}^{2+}\left(\left[\mathrm{Ca}^{2+}\right]_{\text {Mito }}\right)$ inhibitor $\mathrm{Ru} 360$ and mitochondrial ROS inhibitor Mito-TEMPO. MGF and TUDCA effectively reduce PA or Tun-induced ROS increase in RAECs. Meanwhile, $\left[\mathrm{Ca}^{2+}\right]_{\mathrm{ER}}$ 
inhibitor $\mathrm{RuR}$ and $\mathrm{XeC},\left[\mathrm{Ca}^{2+}\right]_{\text {Mito }}$ inhibitor $\mathrm{Ru} 360$ and Mito-TEMPO inhibited ROS generation (Figure 2B).

Mitochondrial ROS inhibitor obviously reduced ROS concentration in cytosolic, which suggested that mitochondria were the primary apparatus for ROS generation. We also tested mitochondrial ROS production using a fluorescence microscope. MGF or TUDCA inhibited mitochondrial ROS increase was stimulated by PA or Tun, meanwhile Ru 360 and Mito-TEMPO also had an inhibitory effect on mitochondrial ROS generation (figure 2C), which suggested that mitochondrial $\mathrm{Ca}^{2+}$ overload induced ROS generation under ER stress, and MGF and TUDCA could reduce ROS generation through attenuating ER stress. We also measured mitochondrial fission by confocal scanning microscopy. MGF, TUDCA, $\left[\mathrm{Ca}^{2+}\right]_{\mathrm{ER}}$ inhibitor RuR and XeC reduced mitochondrial fission induced by PA or Tun (Figure 2E), suggesting that ER stress increased mitochondrial fission. We also detected whether MGF and TUDCA regulated mitochondrial function through testing mitochondrial membrane potential $(\Delta \psi \mathrm{m})$. MGF, TUDCA, and $\left[\mathrm{Ca}^{2+}\right]_{\text {Mito }}$ inhibitor Ru 360 restored PA-induced mitochondrial membrane potential degraded (Figure 2D). These results demonstrated that MGF and TUDCA could inhibit mitochondrial fission and ROS generation caused by ER stress.

\subsection{Independent of Activating AMPK, MGF Inhibited Mitochondrial Fission}

Drp1 is the main factor facilitating mitochondrial fission. It primarily locates in the cytosol at Ser 637 phosphorylation status. If dephosphorylation of Ser 637 occurs, mitochondrial fission will increase. As shown in Figure 3A, the expression of p-Drp1 was reduced by PA or Tun, while MGF and AICAR can regulate its level to prevent cells from undergoing mitochondrial fission. We further investigated the relationship between AMPK and MGF that resulted in inhibited mitochondrial fission. As Figure 3B indicated, MGF also increased p-Drp1 expression after silencing AMPK. The results suggested that MGF did not attenuate mitochondrial fission through AMPK activation.
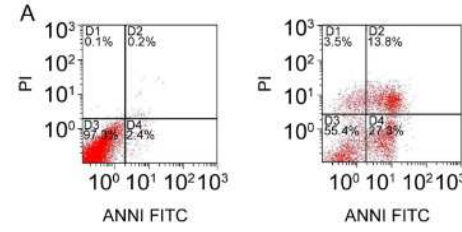

Blank

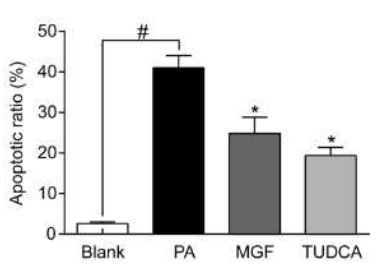

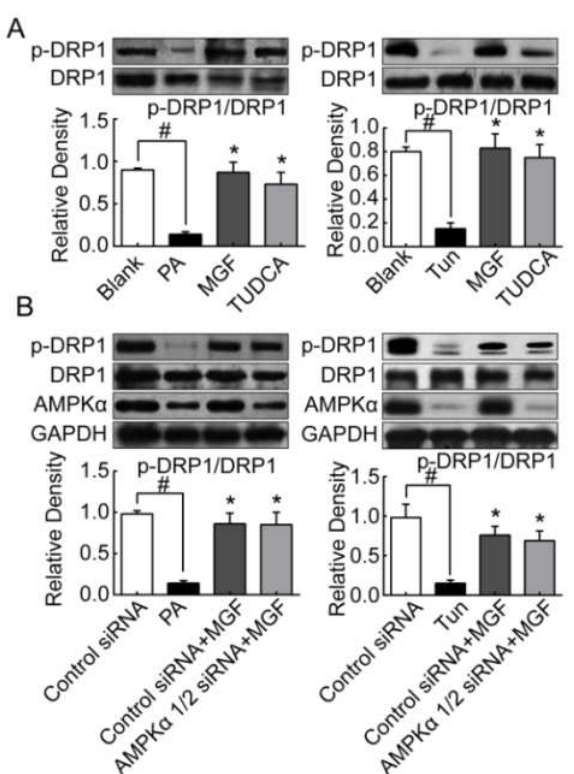

Figure 3. Independent of activating AMPK, MGF inhibited mitochondrial fission. (A): HUVECs were incubated with MGF or TUDCA in the presence of $P A$ and Tun for $24 h$ and $8 h$ respectively; $(B)$ : HUVECs transfected with AMPKa1/2 or control scrambled siRNAs. AMPK and DRP1 phosphorylation were determined by Western blot. The results were expressed as the mean \pm $S D$ of three independent experiments. ${ }^{*} p<0.05$ : indicated group vs PA or Tun only treatment; $\# p<0.05$ : PA or Tun treatment vs untreated control.

\subsection{MGF and TUDCA Inhibited Endothelial Cells Apoptosis}

Caspase-3 is the key factor related to cells apoptosis, so we detected activation of caspase- 3 expression using a commercial Kit. As shown in Figure 4B, MGF and TUDCA reduced PA or Tun-induced caspase- 3 activation in RAECs, and anti-IL-1 $\beta$ neutralizer also inhibited caspase- 3 activation, suggesting that IL-1 $\beta$ was required for caspase-3 activation. After we detected RAECs apoptosis by flow cytometry analysis (Figure 4A), these results conclusively proved that MGF and TUDCA could inhibit cells apoptosis induced by PA.

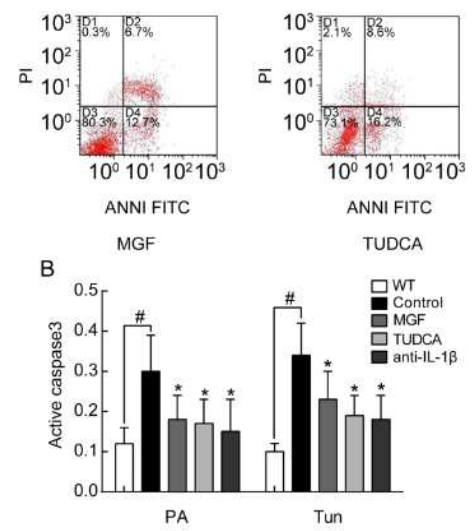

Figure 4. MGF and TUDCA inhibited endothelial cells apoptosis. (A): the apoptosis assay was performed with flow cytometry analysis in RACEs exposed to PA. (B): activated caspase-3 in RAECs, after being exposed to PA and Tun for $24 \mathrm{~h}$ and $8 \mathrm{~h}$ respectively, was determined with a commercial Kit. The result was expressed as the mean $\pm S D$ ( $n=4$ per group). The results were derived from three independent experiments. * $<0.05:$ indicated group vs PA or Tun only treatment; $\# p<0.05$ : PA or Tun treatment vs the untreated control. 


\subsection{MGF and TUDCA Prevented Rat Aorta from PA Stimulated Change of Endothelium-Dependent Vasodilation}

Endothelial dysfunction is accompanied by depression of the endothelium-dependent vasodilation function. Therefore, we stimulated rat aorta with PA and detected endothelial vasodilation function in response to acetylcholine (ACh). The rat aorta was pretreated with MGF and TUDCA for $30 \mathrm{~min}$ and incubated with PA or Tun for $1 \mathrm{~h}$, then fixed in ACh. The results demonstrated that MGF and TUDCA improved endothelial vasodilation function in rat aorta with PA or Tun insult (figure 5C). In vivo, we fed mice with HFD, treated them with MGF and TUDCA, and then measured with fluorescence microscope. As Figure 5A showed, MGF and TUDCA increased NO production response to $\mathrm{ACh}$ in endothelium. We detected the level of NO stimulated by PA in RAECs. RAECs were incubated with MGF and TUDCA for $30 \mathrm{~min}$, exposed to PA for $1 \mathrm{~h}$ and detected with fluorescence microscopy. The results suggested that MGF and TUDCA attenuated PA-induced reduction of NO generation (Figure 5B).
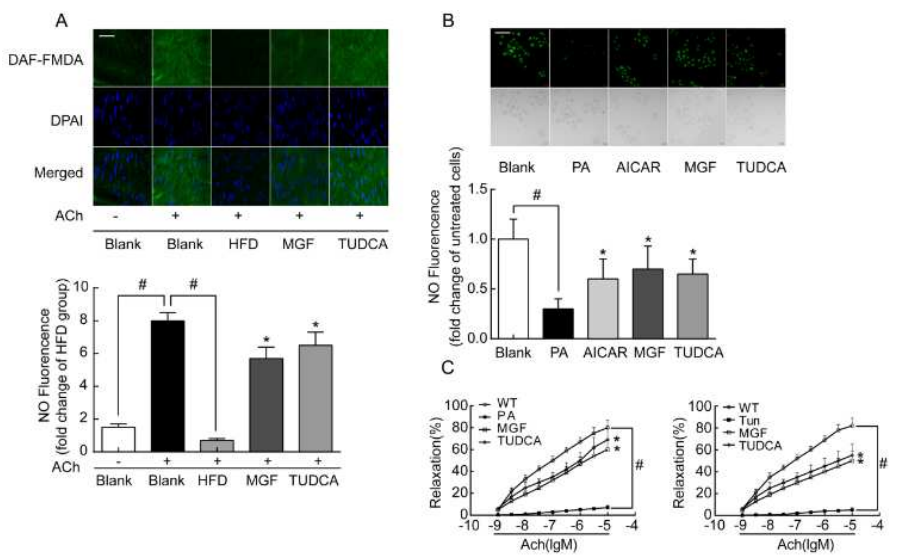

Figure 5. MGF and TUDCA prevented rat aorta from PA stimulated change of endothelium-dependent vasodilation. (A): rat aortas were pretreated with MGF, TUDCA for $30 \mathrm{~min}$ and then stimulated with PA or Tun for $1 \mathrm{~h}$. The data was showed by comparing fold change of fluorescence intensity in WT or MGF or TUDCA treated groups versus HFD group. Scale bar, $50 \mu \mathrm{m}$. (B): RAECs cells were pretreated with MGF or TUDCA for 30 min and then stimulated with PA for $1 \mathrm{~h}$. Intracellular NO was viewed with fluorescence microscopy. Scale bar, $5 \mu \mathrm{m} .(C)$ : the vasodilation was assessed by exposure to ACh. The results were expressed as the mean $\pm S D$ ( $n=6$ per group). The result was for one representative of three independent experiments. * $p<0.05:$ indicated group $v$ S PA or Tun or HFD treatment; \#p<0.05: PA treatment vs untreated control.

\subsection{MGF Ameliorated ER Stress in Aorta Endothelium in HFD-fed Mice}

For testing MGF attenuating ER stress in vivo, mice was provided with HFD for 15 days and treated with MGF (50 $\mathrm{mg} / \mathrm{Kg}$ ) and TUDCA (50 $\mathrm{mg} / \mathrm{Kg})$. Then we detected the protein expression related to ER stress in aorta cells by
Immunohistochemisty. We observed the levels of p-IRE1 and p-PERK were elevated in HFD-fed mice, and Oral administration of MGF and TUDCA reduced p-IRE1 and p-PERK expression, which indicated that MGF and TUDCA could attenuate ER stress in vivo (Figure 6).

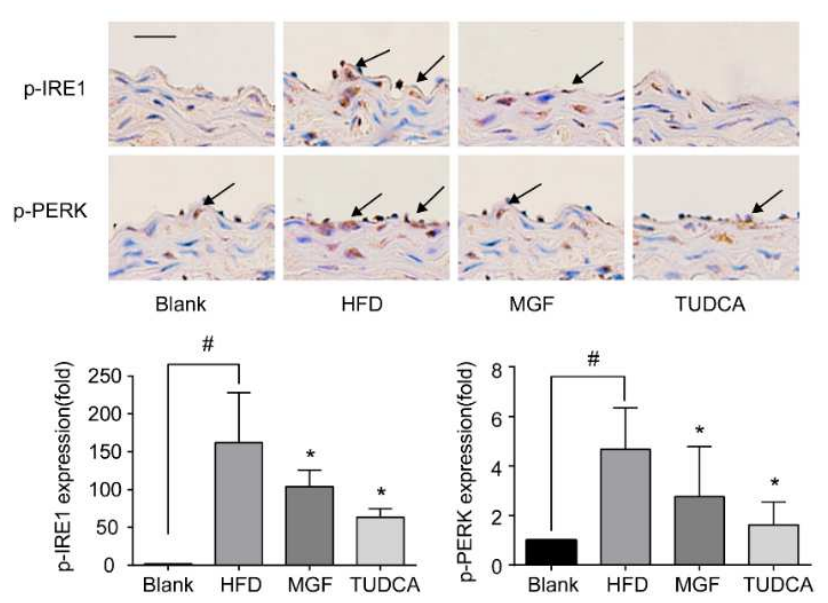

Figure 6. MGF ameliorated ER stress in aorta endothelium in HFD-fed mice. Mice were fed with HFD for 2 weeks. IRE1 and PERK phosphorylations in the mice aorta were viewed by immunohistochemisty staining. Bar Scale $=50 \mu \mathrm{m}$ at $200 \times$ maginification. The result was obtained one from of three different mice. ${ }^{*} p<0.05$ : indicated group vs HFD treatment; $\# p<0.05$ : PA treatment vs untreated control. 


\section{Discussion}

Mangiferin (MGF) can be extracted from the rhizome of Anemarrhena asphodeloides, a fruit used in traditional Chinese Medicine generally used to treat metabolism syndrome, such as type 2 diabetes and insulin resistance. Recent studies have shown that MGF is an AMPK activator, an anti-inflammation and anti-oxidant agent and has a specific function in regulating glucose and lipid metabolism [20, 24-26]. MGF can maintain endothelial homeostasis but its mechanism remains unclear. The results demonstrated that MGF could improve endothelial dysfunction by attenuating $\left[\mathrm{Ca}^{2+}\right]_{\text {mito }}$ overload-induced mitochondrial fission and inhibiting mitochondrial ROS production to depress ER stress and maintain ER homeostasis through activating AMPK pathway.

The endoplasmic reticulum plays a crucial role in maintaining cell homeostasis, which is responsible for protein synthesis, processing, translation, and secretion. It is also important for intracellular calcium homeostasis and lipid biosynthesis [27, 28]. Recently, a variety of studies have demonstrated that ER stress and unfolded protein response (UPR) are associated with chronic metabolic disease and cardiovascular disease [29, 30]. Rachelle E. Kaplon and colleagues demonstrated that endothelial cells in nondiabetic obese adults also undergo ER stress [31], because lipid metabolism dysfunction can disturb ER homeostasis and result in ER stress [27]. In the present study, we used PA stimulated endothelial cells to mimic high blood concentration of FFA in patients with glucose and lipid metabolism disorder. The results elucidated that PA induced ER stress and activated PERK, IRE1 $\alpha$ and ATF4 pathway, while MGF and TUDCA reduced phosphorylation of the protein expression which is relate to ER stress. MGF, as an AMPK activator, detected whether the attenuation of ER stress by MGF required for activating AMPK. The results demonstrated that MGF inhibited ER stress through activating AMPK. Meanwhile, we also used Tun, an ER stress irritant, to test the hypothesis, and the results also proved that MGF inhibited ER stress by activating AMPK.

The mitochondria are responsible for supplying energy to cells and it also participates in cell division, embryonic development, apoptosis and cellular metabolism. In endothelial cells, mitochondrial function is associated with ROS production and calcium balance. Many studies have elucidated that damaged mitochondrial function is related to endothelial dysfunction [32]. In the present study, the endothelial cells was treated with PA to increase mitochondrial fission through dephosphorylating Drp1 at Ser 637 residue, and demonstrated that MGF could reduce mitochondrial fission by improving Drp1 phosphorylation. Drp1, a mitochondrial fission dynamic related protein, is an important role in mitochondrial fission process. Normally, it exists in cytoplasm in phosphorylated status, induced by cell stress, and then it recruits at the Outer Mitochondrial Membrane (OMM) to trigger mitochondrial fission process
$[33,34]$. Generally, there is a dynamic balance between mitochondrial fission and fusion to maintain mitochondrial function, but stress can disturb this balance and then trigger mitochondrial apoptosis or autophagy. The mechanism of PA-induced mitochondrial fission is related to calcium homeostasis. Mitochondria, an $\mathrm{Ca}^{2+}$ buffer organelle, plays a crucial role in maintaining calcium homeostasis in cytoplasm. In ER stress conditions, a large flux of $\mathrm{Ca}^{2+}$ transfers from ER to cytoplasm, and then mitochondria takes in a large amount of $\mathrm{Ca}^{2+}$ to maintain calcium homeostasis in cytoplasm, and finally results in $\mathrm{Ca}^{2+}$ overload in mitochondria [35]. Mitochondrial $\mathrm{Ca}^{2+}$ overload leads to mitochondria dysfunction, destroyed mitochondrial respiratory chain function and production of a high ROS concentration. In turn ROS further facilitates mitochondrial fission and induces mitochondria to release pro-apoptosis factor. After that, the cells undergo an apoptosis process [36].

We hypothesized that the mechanism of MGF inhibiting mitochondrial fission was associated with inhibiting ER stress. The results suggested that ER stress significantly improved mitochondrial fission in endothelial cells and pretreatment with MGF and TUDCA could reduce mitochondrial fission, therefore, MGF attenuated mitochondrial fission by inhibiting ER stress. Mitochondrial dysfunction induced metabolism disorder of endothelial cells and then influenced endothelial function. The endothelial function was detected by endothelium-dependent vasodilation. NO was shown to be related to endothelial vasodilation function in rat aorta endothelium-dependent experiment, and the results demonstrated that MGF could improve PA-induced decreasing of endothelial vasodilation. In the present study, the results demonstrated that protein expression related to ER stress was increased in aorta endothelial cells of HFD-fed mice, which facilitated mitochondrial fission, while MGF could attenuate ER stress and mitochondrial fission. However, a recent report has discovered that the AMPK participates in the mitochondrial fission process and AMPK activator increases mitochondrial fission [37].

AMPK, as a sensor of cellular energy, plays a crucial role in cell metabolism, survival and death and also regulates metabolic balance within the entire body [38, 39]. Recent studies have shown that AMPK plays a crucial role in inhibiting metabolism disease, such as diabetes, obesity and cardiovascular disease [40]. There are various influence factors in the AMPK pathway, such as calcium concentration and the ratio of AMP/ATP or ADP/ATP. Several researches have elucidated that many plant extract can activate AMPK, but the mechanisms are different [41]. The classical medicine metformin activated AMPK through the inhibition of the mitochondrial respiratory chain to reduce ATP synthesis [42], but the mechanism of MGF activating AMPK is unclear. Pasha Apontes et.al. reported that MGF can improve glucose metabolism to prevent against metabolism disorder induced by HFD, an effect that is similar to the activation of AMPK thereby ameliorating metabolism dysfunction [21]. 


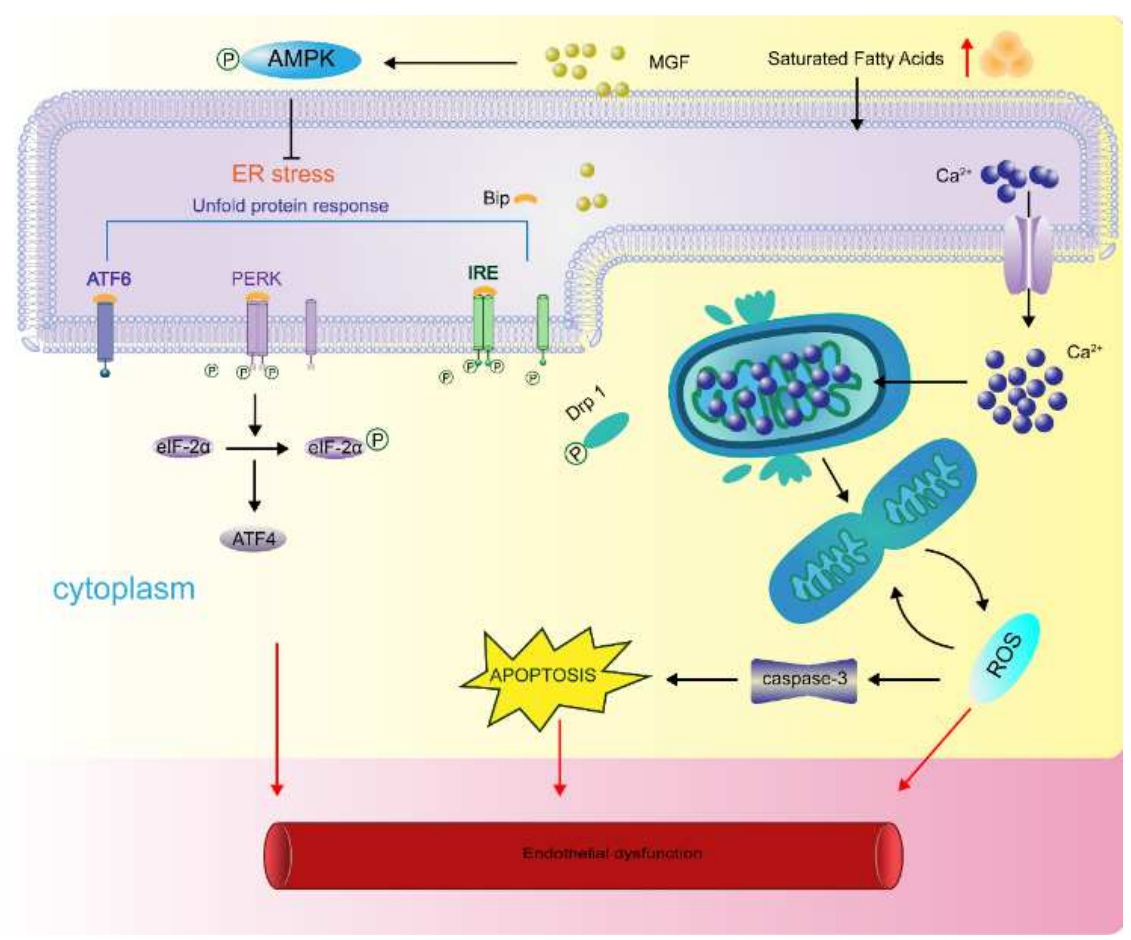

Figure 7. The mechanism of MGF attenuating endothelial dysfunction. After being stimulated by Saturated Fatty Acid for a long time, ER stress occurs and induces the exudation of $\mathrm{Ca}^{2+}$ from ER lumen to cytoplasm. Mitochondria could take in a large amount of Ca ${ }^{2+}$ to build a normal concentration gradient in cytoplasm. Mitochondrial $\mathrm{Ca}^{2+}$ overload induced ROS generation in mitochondria, and then ROS increased mitochondria fission and releasing of apoptosis factor which triggers the apoptosis program of cells. Apoptosis, ER stress and ROS can induce endothelial dysfunction. MGF could attenuate ER stress through activating AMPK and reduce mitochondrial fission and ROS generation to improve endothelial cells function.

In conclusion, the mechanism of MGF attenuating endothelial dysfunction is closely related to the activation of AMPK which inhibits ER stress. ER plays a vital role in maintaining cell homeostasis and PA can be used to break its normal ER function. Under ER stress, excess $\mathrm{Ca}^{2+}$ exudate from ER lumen to cytoplasm [9, 35], mitochondria could take in a large amount of $\mathrm{Ca}^{2+}$ to build a normal concentration gradient in cytoplasm. Mitochondrial $\mathrm{Ca}^{2+}$ overload induced ROS generation in mitochondria, and then ROS increased mitochondria fission which released the apoptosis factor thereby triggering the apoptosis program of cells. AMPK, as a cell energy senor, could be activated by stress stimulation. If stress is presented for a long time, it is difficult for AMPK to maintain the balance of cell metabolism. Chronic metabolic diseases, like diabetes and obesity, tend to cause long-term stress in the whole body, so we need medical treatment to help the body maintain normal physiological function. Diabetes and obesity always accompany hyperlipidermia, PA was used to create the environment similar to hyperlididermia. This present experiment demonstrated the mechanism of MGF maintaining endothelial homeostasis through inhibiting ER stress and mitochondrial fission. The results should significantly contribute to the development of medicine, such as MGF, in treating cardiovascular metabolism disease.

\section{Acknowledgements}

This work was supported by the Qing Lan Project of Jiangsu Province (2014). Y. C. appreciated Cuiping Kuai for assistance with manuscript writing.

\section{References}

[1] Van Gaal LF, Mertens IL, De Block CE. Mechanisms linking obesity with cardiovascular disease [J]. NATURE, 2006, 444(7121): 875-880.

[2] Kaplon RE, Chung E, Reese L, et al. Activation of the Unfolded Protein Response in Vascular Endothelial Cells of Nondiabetic Obese Adults [J]. The Journal of Clinical Endocrinology \& Metabolism, 2013, 98(9): E1505-E1509.

[3] Appleton SL, Seaborn CJ, Visvanathan R, et al. Diabetes and Cardiovascular Disease Outcomes in the Metabolically Healthy Obese Phenotype: A cohort study [J]. DIABETES CARE, 2013, 36(8): 2388-2394.

[4] Despres JP. Body Fat Distribution and Risk of Cardiovascular Disease: An Update [J]. CIRCULATION, 2012, 126(10): 1301-1313.

[5] Cai H, Harrison DG. Endothelial dysfunction in cardiovascular diseases: the role of oxidant stress [J]. CIRC RES, 2000.

[6] Thomas Heitzer M, Titus Schlinzig B, Karoline Krohn B, et al. Endothelial Dysfunction, Oxidative Stress, and Risk of Cardiovascular Events in Patients With Coronary Artery Disease [J]. CIRCULATION, 2001, 104: 2673-2678.

[7] Chambers JE, Marciniak SJ. Cellular Mechanisms of Endoplasmic Reticulum Stress Signaling in Health and Disease. 2. Protein misfolding and ER stress [J]. AJP: Cell Physiology, 2014, 307(8): C657-C670. 
[8] Fu S, Watkins SM, Hotamisligil GKS. The Role of Endoplasmic Reticulum in Hepatic Lipid Homeostasis and Stress Signaling [J]. CELL METAB, 2012, 15(5): 623-634.

[9] Sovolyova N, Healy S, Samali A, et al. Stressed to death mechanisms of ER stress-induced cell death [J]. BIOL CHEM, 2014, 395(1): 1-13

[10] Hall AR, Burke N, Dongworth RK, et al. Mitochondrial fusion and fission proteins: novel therapeutic targets for combating cardiovascular disease [J]. BRIT J PHARMACOL, 2014, 171(8): 1890-1906.

[11] Orrenius S, Gogvadze V, Zhivotovsky B. Calcium and mitochondria in the regulation of cell death [J]. BIOCHEM BIOPH RES CO, 2015, 460(1): 72-81.

[12] Contreras L, Drago I, Zampese E, et al. Mitochondria: The calcium connection [J]. Biochimica et Biophysica Acta (BBA) - Bioenergetics, 2010, 1797(6-7): 607-618.

[13] Pangare M, Makino A. Mitochondrial function in vascular endothelial cell in diabetes [J]. J. Smooth Muscle Res., 2012, 48(6): 1-26.

[14] Pharmacol JC. Hyperglycemic Conditions Affect Shape and $\mathrm{Ca}^{2+}$ Homeostasis of Mitochondria in Endothelial Cells [J]. J Cardiovasc Pharmacol, 2004, 44: 423-436.

[15] Dai DF, Rabinovitch PS, Ungvari Z. Mitochondria and Cardiovascular Aging [J]. CIRC RES, 2012, 110(8): $1109-1124$.

[16] Esper RJ, Nordaby RA, Vilariño JO, et al. Endothelial dysfunction: a comprehensive appraisal [J]. CARDIOVASC DIABETOL, 2006, 5: 4 .

[17] Pinton P, Giorgi C, Siviero R, et al. Calcium and apoptosis: ER-mitochondria $\mathrm{Ca}^{2+}$ transfer in the control of apoptosis [J]. ONCOGENE, 2008, 27(50): 6407-6418.

[18] Kluck RM, Bossy-Wetzel E, Green DR, et al. The Release of Cytochrome c from Mitochondria: A Primary Site for Bcl-2 Regulation of Apoptosis [J]. SCIENCE, 1997, 275(5303): 1132-1136.

[19] Zhou L, Pan Y, Chonan R, et al. Mitigation of Insulin Resistance by Mangiferin in a Rat Model of Fructose-Induced Metabolic Syndrome Is Associated with Modulation of CD36 Redistribution in the Skeletal Muscle [J]. J PHARMACOL EXP THER, 2015, 356(1): 74-84.

[20] Song J, Li J, Hou F, et al. Mangiferin inhibits endoplasmic reticulum stress-associated thioredoxin-interacting protein/NLRP3 inflammasome activation with regulation of AMPK in endothelial cells [J]. METABOLISM, 2015, 64(3): 428-437.

[21] Apontes P, Liu Z, Su K, et al. Mangiferin Stimulates Carbohydrate Oxidation and Protects Against Metabolic Disorders Induced by High-Fat Diets [J]. DIABETES, 2014, 63(11): 3626-3636.

[22] Li J, Wang Y, Wang Y, et al. Pharmacological activation of AMPK prevents Drpl-mediated mitochondrial fission and alleviates endoplasmic reticulum stress-associated endothelial dysfunction [J]. J MOL CELL CARDIOL, 2015, 86: 62-74.

[23] Toyama EQ, Herzig S, Courchet J, et al. Metabolism. AMP-activated protein kinase mediates mitochondrial fission in response to energy stress [J]. SCIENCE, 2016, 351(6270):
$275-281$.

[24] Niu Y, Li S, Na L, et al. Mangiferin Decreases Plasma Free Fatty Acids through Promoting Its Catabolism in Liver by Activation of AMPK [J]. PLOS ONE, 2012.

[25] Sellamuthu PS, Arulselvan P, Muniappan BP, et al. Mangiferin fromSalacia chinensis Prevents Oxidative Stress and Protects Pancreatic $\beta$-Cells in Streptozotocin-Induced Diabetic Rats [J]. J MED FOOD, 2013, 16(8): 719-727.

[26] Tsubaki M, Takeda T, Kino T, et al. Mangiferin suppresses CIA by suppressing the expression of TNF- $\alpha$, IL- 6 , IL- $1 \beta$, and RANKL through inhibiting the activation of NF- $\kappa \mathrm{B}$ and ERK1/2 [J]. Am J Transl Res., 2015, 7(8): 1371-1381.

[27] Go Khan S. Hotamisligil. Endoplasmic Reticulum Stress and the Inflammatory Basis of Metabolic Disease [J]. CELL, 2010, 140(6): 900-917.

[28] Özcan U, Cao Q, Erkan Yilmaz, et al. Endoplasmic Reticulum Stress Links Obesity, Insulin Action, and Type 2 Diabetes [J]. Science, 2004, 306(5695): 457-461.

[29] Minamino T, Komuro I, Kitakaze M. Endoplasmic Reticulum Stress As a Therapeutic Target in Cardiovascular Disease [J]. CIRC RES, 2010, 107(9): 1071-1082.

[30] Basha B, Samuel SM, Triggle CR, et al. Endothelial Dysfunction in Diabetes Mellitus: Possible Involvement of Endoplasmic Reticulum Stress? [J]. Experimental Diabetes Research, 2012, 2012: 1-14.

[31] Kaplon RE, Chung E, Reese L, et al. Activation of the Unfolded Protein Response in Vascular Endothelial Cells of Nondiabetic Obese Adults [J]. The Journal of Clinical Endocrinology \& Metabolism, 2013, 98(9): E1505-E1509.

[32] Shenouda SM, Widlansky ME, Chen K, et al. Altered Mitochondrial Dynamics Contributes to Endothelial Dysfunction in Diabetes Mellitus [J]. CIRCULATION, 2011.

[33] Chang C, Blackstone C. Dynamic regulation of mitochondrial fission through modification of the dynamin-related protein Drp1 [J]. ANN NY ACAD SCI, 2010, 1201(1): 34-39.

[34] Lee Y, Jeong S, Karbowski M, et al. Roles of the Mammalian Mitochondrial Fission and Fusion Mediators Fis1, Drp1, and Opal in Apoptosis [J]. MOL BIOL CELL, 2004, 15(11): 5001-5011.

[35] Bianchi K, Rimessi A, Prandini A, et al. Calcium and mitochondria: mechanisms and functions of a troubled relationship [J]. Biochimica et Biophysica Acta (BBA) Molecular Cell Research, 2004, 1742(1-3): 119-131.

[36] Yu T, Sheu SS, Robotham JL, et al. Mitochondrial fission mediates high glucose-induced cell death through elevated production of reactive oxygen species [J]. CARDIOVASC RES, 2008, 79(2): 341-351.

[37] Huang F, Liu K, Du H, et al. Puerarin attenuates endothelial insulin resistance through inhibition of inflammatory response in an IKK $\beta /$ IRS-1-dependent manner [J]. BIOCHIMIE, 2012, 94(5): 1143-1150.

[38] Okoshi R, Ozaki T, Yamamoto $\mathrm{H}$, et al. Activation of AMP-activated Protein Kinase Induces p53-dependent Apoptotic Cell Death in Response to Energetic Stress [J]. J BIOL CHEM, 2008, 283(7): 3979-3987. 
[39] Hardie DG, Ross FA, Hawley SA. AMPK: a nutrient and energy sensor that maintains energy homeostasis [J]. NAT REV MOL CELL BIO, 2012, 13(4): 251-262.

[40] Saha A, Coughlan K, Valentine R, et al. AMPK activation: a therapeutic target for type 2 diabetes?[J]. Diabetes, Metabolic Syndrome and Obesity: Targets and Therapy, 2014, 7: 241-253.
[41] Langendorf CG, Kemp BE. Choreography of AMPK activation [J]. CELL RES, 2014, 25(1): 5-6.

[42] Miller RA, Birnbaum MJ. An energetic tale of AMPK-independent effects of metformin [J]. J CLIN INVEST, 2010, 120(7): 2267-2270. 